\title{
The effects of model climate bias on ENSO variability and ensemble prediction.
}

$\begin{array}{lll}\text { S. L. Osbrough } & & \text { J. S. Frederiksen } \\ & & \text { C. S. Frederiksen } \\ & \end{array}$

(Received 6 March 2019; revised 10 October 2019)

\begin{abstract}
New methods are presented for determining the role of coupled ocean-atmosphere model climate bias on the strength and variability of the El Niño-Southern Oscillation (ENSO) and on the seasonal ensemble prediction of El Niño and La Niña events. An intermediate complexity model with a global atmosphere coupled to a Pacific basin ocean is executed with parallelised algorithms to produce computationally efficient year-long forecasts of large ensembles of coupled flow fields, beginning every month between 1980 and 1999. Firstly, the model is provided with forcing functions that reproduce the average annual cycle of climatology of the atmosphere and ocean based on reanalysed observations. We also configure the model to generate realistic ENSO fluctuations. Next, an ensemble prediction scheme is employed which produces perturbations that amplify rapidly over a month. These
\end{abstract}

DOI:10.21914/anziamj.v60i0.14092 gives this article, (c) Austral. Mathematical Soc. 2019. Published October 18, 2019, as part of the Proceedings of the 18th Biennial Computational Techniques and Applications Conference. ISSN 1445-8810. (Print two pages per sheet of paper.) Copies of this article must not be made otherwise available on the internet; instead link directly to the DOI for this article. 
perturbations are added to the analyses and give the initial conditions for the ensemble forecasts. The skill of the forecasts is presented and the dependency on the annual and ENSO cycles determined. Secondly, we replace the forcing functions in our model with functions that reproduce the averaged annual cycles of climatology of two state of the art, comprehensive Coupled General Circulation Models. The changes in skill of subsequent ensemble forecasts elucidate the roles of model bias in error growth and potential predictability.

\section{Contents}

1 Introduction

2 Model configuration and forcing functions

3 Experimental design

4 Theory

$\mathrm{C} 220$

5 Results

$\mathrm{C} 220$

5.1 Growth rates . . . . . . . . . . . . . . .

5.2 Analysis runs

5.3 Forecasts. . .

6 Climate biases in ACCESS1-3 and CCSM4

C225

7 Conclusion

$\mathrm{C} 226$

\section{Introduction}

El Niño-Southern Oscillation (ENSO) is a natural cycle of changing Pacific Ocean temperature, circulation and atmospheric winds that influences ex- 
treme Australian climatic conditions over seasonal to interannual time scales. Coupled General Circulation Models (CGCMs) used for prediction studies are limited by initial conditions and their representation of physical processes. These contain systematic climate biases that make modelling the diverse behaviour and evolution of ENSO a challenging problem. Here, we are interested in determining the role of coupled ocean-atmosphere model climate bias on the strength and variability of ENSO and on the seasonal ensemble prediction of El Niño and La Niña events. We do this by replacing the forcing functions in our efficient model by functions that reproduce the average annual cycle of climatologies of two CGCMs.

Section 2 describes the model and the datasets required for determining the forcing functions. Section 3 outlines the experimental method and Section 4 describes the equations used to calculate the growth rates of perturbations and their relative amplification factor. In Section 5 we discuss the behaviour of perturbation growth, the ENSO variability found in analysis runs and the seasonal variability of the average error during the 1980-1999 forecasts.

\section{Model configuration and forcing functions}

The Primitive Equation Coupled Ocean and Atmosphere Model (PECOAM) used in this study was developed for its code useability, computational efficiency and predictive skill in producing forecasts of ENSO events [2, 1, 5, 4]. These benefits are due to the efficient dynamics and physics used for coupling upper and lower levels of the ocean at $50 \mathrm{~m}$ and $150 \mathrm{~m}$ with atmosphere at $250 \mathrm{hPa}$ and $750 \mathrm{hPa}$, respectively.

The global atmospheric component of the model is based on the primitive equations on a sphere coupled through surface wind stresses and heat fluxes to a Pacific basin ocean. Model resolution is $2.3^{\circ}$ latitude by $3.75^{\circ}$ longitude, which is sufficient for our purposes in investigating the variability and predictability of ENSO. Frederiksen, Frederiksen and Balgovind [2] provide full 
details on model structure and parameters.

The monthly observed sea surface temperatures (SSTs) used for this experiment were taken from the period 1980 to 2000 with the wind stresses and temperatures sourced from the National Centers for Environmental Prediction/National Center for Atmospheric Research (NCEP/NCAR) Reanalysis (NNR) data set [8]. This configuration of the PECOAM, as described by Frederiksen, Frederiksen and Balgovind [2], determines our control case and is referred to here as the 'Standard' model. For the same time period we also use corresponding model data sets from the NCAR Community Climate System Model (CCSM4) [6], as well as the Commonwealth Scientific and Industrial Research Organisation (CSIRO) and Bureau of Meteorology's (BOM) Australian Community Climate and Earth-System Simulator (ACCESs1-3) [9]. The CGCM model output used in this study was sourced from The World Climate Research Program's Working Group on Coupled Modelling fifth phase of the Coupled Model Intercomparison Project (CMIP5) [10].

We are interested in simulating past major ENSO events using our primitive equation model beginning 1 January 1980. The subsequent twenty year period chosen for analysis includes the El Niños of 1982-83, 1987-88 and 1997-98 and La Niñas of 1983-85, 1988-89 and 1998-2000.

\section{$3 \quad$ Experimental design}

For this study we begin by forcing our PECOAM with the observed SSTs, NNR wind stresses and thermal forcing functions to simulate the average observed annual cycle of climatology of the atmosphere and ocean. The model is also configured to generate realistic ENSO fluctuations, as explained by Frederiksen, Frederiksen and Balgovind [1].

Next, we use the model forced with NNR forcings to conduct a control forecast from the analysis. Perturbations are then bred and added and subtracted to the analysis to create multiple paired member ensemble forecasts. Frederiksen, 
Frederiksen and Osbrough [5, 4], and references therein, showed that as forecasts progress in time, the ensemble mean is a better prediction than a single control forecast. The initial ensemble perturbations generated must display realistic growth rates that reflect the dynamic property of error growth in the large scale coupled modes of the ocean and atmosphere. For this reason, perturbations are grown over a time period of one month. The method chosen for generating the perturbations is the cyclic mode method [5, 4]. Given our constraints on resources, the optimal choice to produce suitable ensemble members is eight paired cyclic ensemble perturbations (with the same structures but different signs) executed in parallel, with breeding cycles iterated three times for each month. These perturbations were added to the analyses to provide the initial conditions for the twelve month ensemble forecasts beginning 1 January 1980.

Next, we repeat our experiment replacing observed SST, wind stress and thermal forcings with data taken from two selected general circulation models, and compare the skill of subsequent forecasts. Our choice of CGCMs, namely ACCESS1-3 and CCSM4, was decided on each model's ability to reproduce large scale dynamics of the Southern Hemispheric atmospheric circulation and reproduce 20th century trends in baroclinicity [3, 7].

Forcing functions are provided to PECOAM which produces eight member ensembles of twelve month forecasts, every three days or every month, for twenty years from each of NNR, ACCESS1-3 and CCSM4. Because of the chaotic nature of the coupled ocean-atmosphere system, it is not expected that the timing of ENSO events simulated in the PECOAM using CGCM forcings will be coincident with those simulations forced by the NNR; however we examine if the frequency and amplitude broadly correspond. The ACCESS1-3 analyses are perturbed towards CCSM 4 atmospheric climatologies to also examine the role of external climate drift. The theory of growth rates and amplification factors used to calculate the seasonal variability and magnitude of cyclic modes is discussed in the next section. 


\section{Theory}

Let $x(\tau, t)$ be the vector of grid point values of any of the atmospheric or oceanic fields of a cyclic mode initiated at time $t$ and evolved to time $t+\tau$. The total amplification factor $A(\tau, t)$ of an evolved perturbation is the ratio of the root mean square (RMS) of our vector of grid point values at the evolved time $t+\tau$ and the initial time $t$ [5]. The local total growth rate averaged over a 30 day interval $T_{30}$ is then calculated by

$$
\tilde{\omega}_{i}(t)=\frac{1}{T_{30}} \ln A\left(T_{30}, t\right),
$$

and the global growth rate is the average of the local total growth rate $\omega_{i}=\left\langle\tilde{\omega}_{i}(t)\right\rangle$ for $t$ ranging between 0 and $T_{\max }$ where $T_{\max }$ is the last initial time of the forecast. The local relative growth rate is then $\omega_{i}(t)=\tilde{\omega}_{i}(t)-\omega_{i}$. Knowing these relationships we now derive the relative amplification factor

$$
R(t)=\exp \int_{0}^{t} \widehat{\omega}_{i}(s) d s .
$$

\section{Results}

\subsection{Growth rates}

We now compare the average local total growth rates and relative amplification factors of the coupled ocean-atmosphere instability fields obtained by the cyclic method of breeding for a month starting every three days using the PECOAM with Standard, ACCESS1-3 and CCSM4 forcing functions. Figure 1 shows the growth characteristics of cyclic mode fields for the potential temperature in the atmosphere and temperature in the ocean.

The PECOAM forecast runs with CCSM4 and ACCESS1-3 forcings exhibit similar growth characteristics to those of the Standard PECOAM run. Figure 1(a) 
Growth Rates
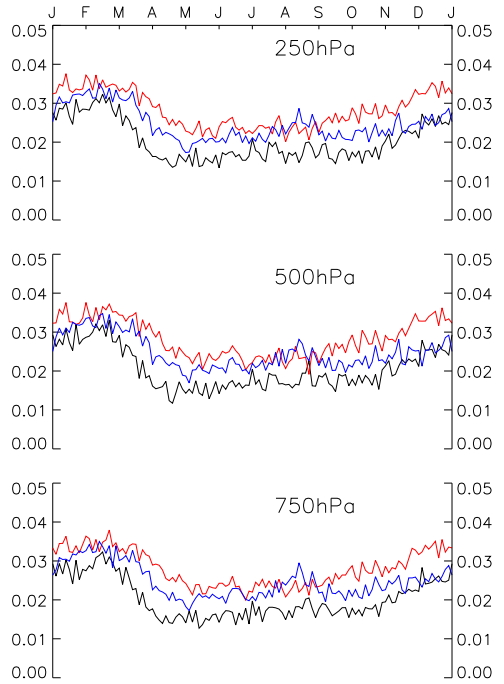

(a)
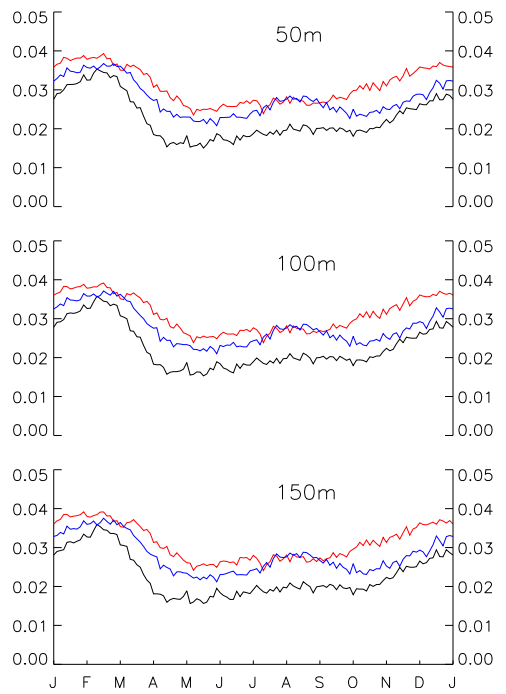

Relative Amplification Factor
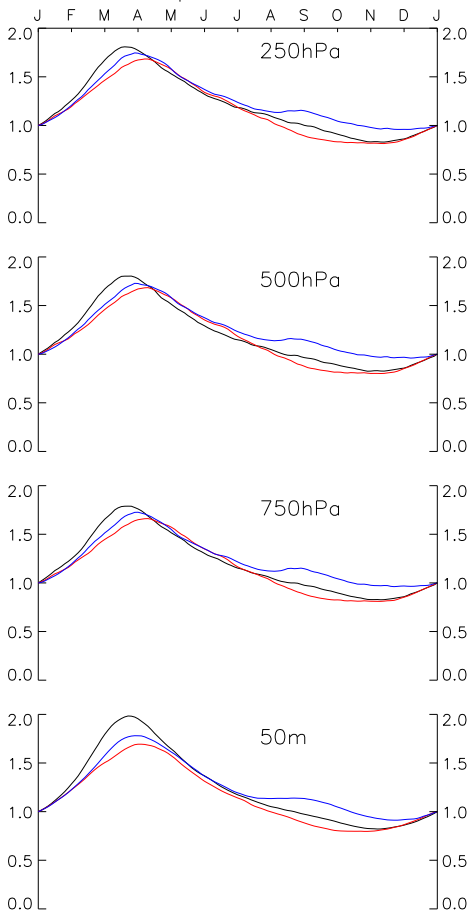

(b)
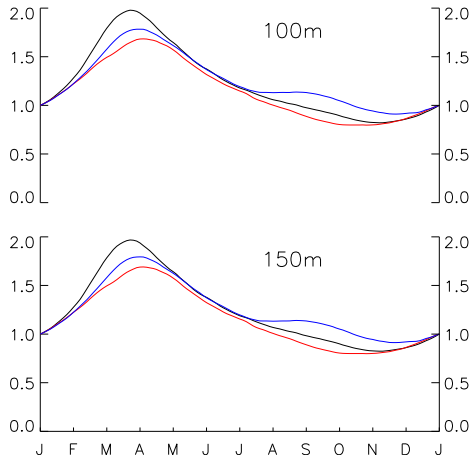

Figure 1: (a) Cyclic mode growth rates per day and (b) relative amplification factors (non dimensional) for: (top three) temperature at different atmospheric pressures; and (bottom three) ocean depths, averaged over the period 1980 to 1999. Coloured lines show PECOAM ensemble forecast runs with forcing functions: (black) Standard; (red) ACCESs1-3; and (blue) CCSM4. 
shows that the modes in the atmosphere and ocean have very similar growth properties over the annual cycle, which is characteristic of coupled modes. The cyclic modes at the different levels in both the ocean and atmosphere have growth rates of similar magnitude and in all cases the maximum growth rate precedes the relative amplification factor maximum in early boreal spring. Contrary to the results from PECOAM forced with ACCESS1-3, the simulations with CCSM4 forcings produce a secondary maximum in the growth rates preceding the relative amplification factor maximum in September.

Because of perturbed analyses, the growth rates from the simulation with ACCESS1-3 forcings have errors that grow overall much faster than those of either the Standard run or CCSM4 forced PECOAM runs. The average global growth rates of the cyclic modes across the levels are $0.0215,0.0295$ and 0.0263 per day for the PECOAM runs with Standard, ACCESS1-3 and CCSM4 forcings, respectively. Errors grow faster than expected, with ACCESS1-3 and CCSM4 forcings resulting in e-folding times of 34 and 38 days, respectively, as opposed to the 47 days produced with the Standard forcings. This result suggests that CCSM4 has a more unstable basic state than the Standard run and would perform less reliably in seasonal forecasting of large scale disturbances in the equatorial region.

In Figure 1(b) the similarities in the seasonal cycles of the relative amplification factors of the simulations are seen, with maximum values occurring in early boreal spring and then falling away in late boreal spring to low values in boreal autumn, followed by a rise in values during winter. However, there are notable differences between the relative amplification factors with Standard and CGCM forcings. The ACCESS1-3 forced runs have closer minimum values to those of the Standard forced runs, and the CCSM 4 forced results show a secondary maxima in September for all atmosphere potential temperature and ocean temperature levels. 


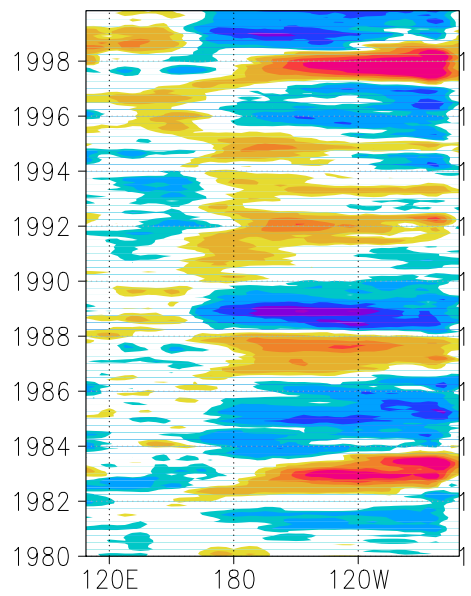

(a) Standard

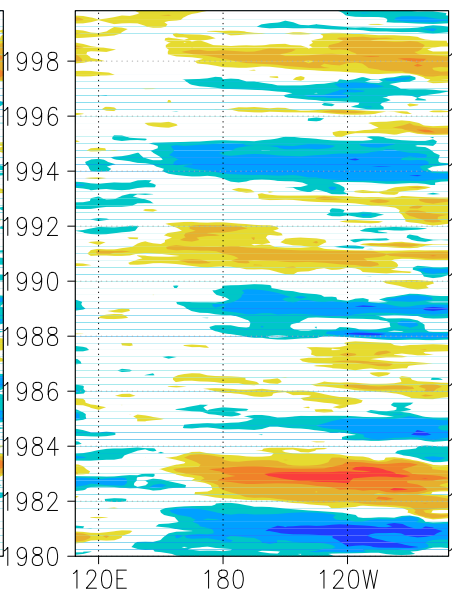

(b) ACCESS1-3

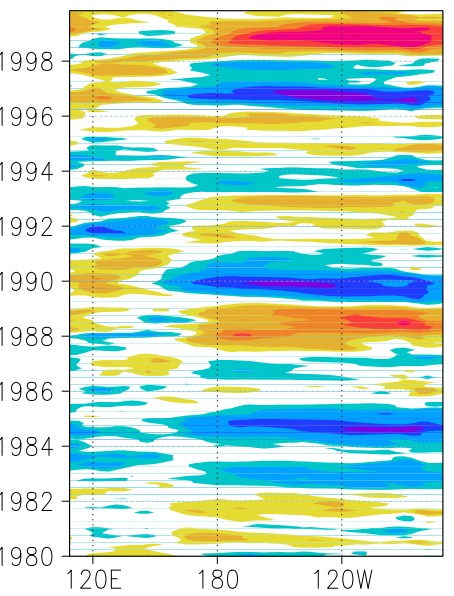

(c) $\operatorname{CCSM} 4$

Figure 2: Ocean temperature anomalies $(\mathrm{K})$ at $50 \mathrm{~m}$, averaged over $5^{\circ} \mathrm{S}-5^{\circ} \mathrm{N}$ for analysis runs from 1980 to 2000 with: (a) Standard; (b) ACCESs1-3; and (c) CCSM4 forcings.

\subsection{Analysis runs}

Figure 2 depicts Hovmöller diagrams showing $50 \mathrm{~m}$ ocean temperature anomalies averaged over $5^{\circ} \mathrm{S}$ to $5^{\circ} \mathrm{N}$ latitude for each month of the twenty year analysis run beginning 1 January 1980. Positive warm ocean anomalies are shown in red and are indicative of the occurrence of El Niño events in the eastern Pacific. Conversely, negative cold ocean anomalies are shown in blue and are indicators of La Niña events.

The Standard model analysis run in Figure 2(a) captures the observed ocean temperatures including those of the major El Niño and La Niña events summarised in Section 2. The peak temperature anomalies are centred between approximately $160^{\circ} \mathrm{W}$ and $80^{\circ} \mathrm{W}$ and correspond with the location of these major events in the eastern Pacific. Both runs with ACCESS1-3 and 
CCSM4 forcings display reasonable frequencies of events at similar longitudes, yet both models largely underestimate the magnitude of ocean temperature anomalies, with ACCESS1-3 forcings giving peaks less than half of the observed.

\section{$5.3 \quad$ Forecasts}

Yearly forecasts are conducted and initialised with the analysis data starting at every month between 1980 and 1999 for each of the Standard, ACCESS1-3 and CCSM 4 forcings. Figure 3 compares the yearly average forecast RMS errors for the control, ensemble and the control minus the ensemble difference.

When we compare results based on PECOAM forced by ACCESS1-3 or CCSM4 with the Standard forced run, we see similarities in the RMS forecast errors, with errors for ACCESS1-3 being larger because of external climate drift. In general, forecast errors increase with time until the boreal spring predictability barrier (BSPB) in March-April-May, after which errors reduce [5]. In addition, errors observed for forecasts initiated at the end of boreal spring have increased forecast error. In each case, the ensemble outperforms the control forecast as indicated by the positive values in the difference plots shown in Figures $3(\mathrm{c}, \mathrm{f}, \mathrm{i})$. The majority of improvement from using an ensemble of forecast runs occurs at the time of the BSPB or towards the end of a twelve month forecast. With the CCSM4 forcing function we see an additional error peak in forecasts initiated during boreal spring, as is seen with the Standard forced run. Again, similar to the Standard forced run, the CGCM forcing functions also result in improvements in ensemble forecasts run during March-April-May, depicting an improvement in error reduction for forecasts conducted during the period of BSPB [4]. 

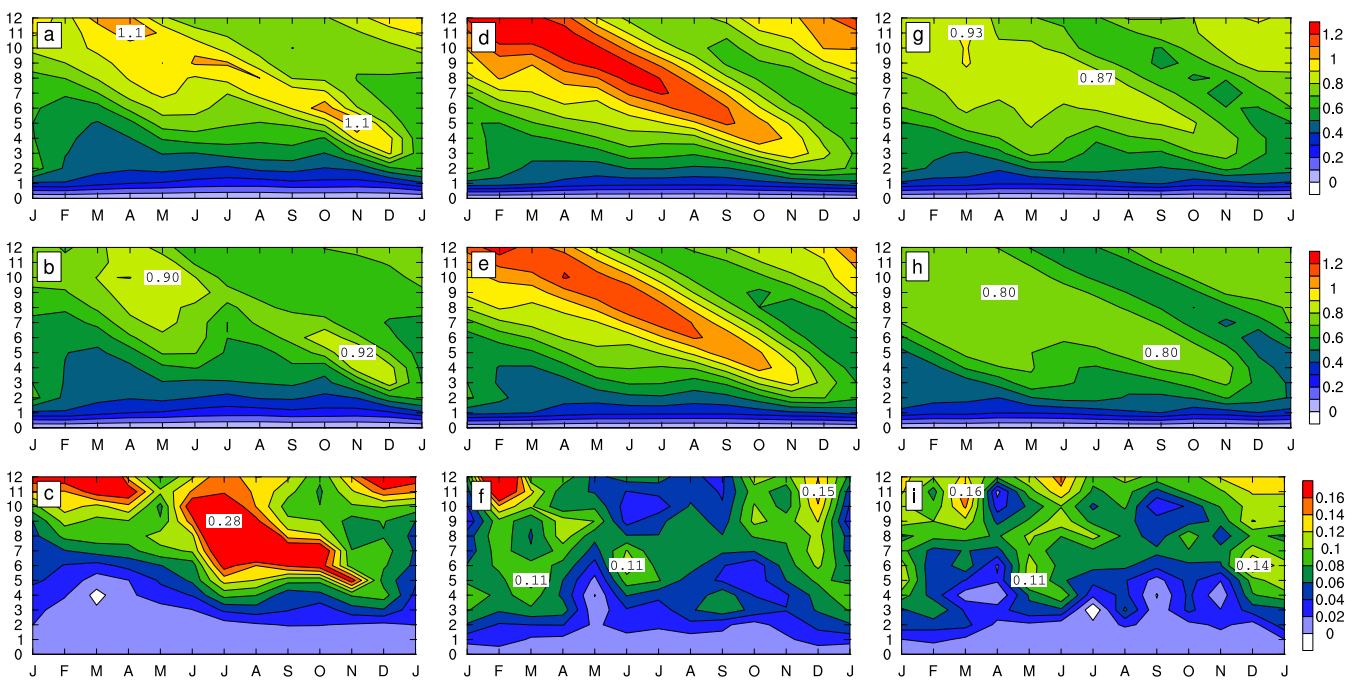

Figure 3: Monthly averaged RMS errors of forecast of $50 \mathrm{~m}$ ocean temperatures in the $\mathrm{NIN} O 3+$ region (defined as $90^{\circ} \mathrm{W}$ to $150^{\circ} \mathrm{W}$ longitude and $10^{\circ} \mathrm{S}$ to $10^{\circ} \mathrm{N}$ latitude) as functions of forecast month for forecasts over all years between January 1980 to December 1999 for: (top) control; (centre) ensemble mean (based on eight cyclic mode ensemble members); and (bottom) control minus the ensemble mean. The PECOAM runs use forcing functions: (a)-(c) Standard; (d)-(f) ACCESS1-3; and (g)-(i) CCSM4. Tick intervals are number of months of forecast.

\section{Climate biases in ACCESS1-3 and CCSM4}

All coupled CGCMs used in seasonal forecasting produce error growth affected by climate biases within the model. There are significant sources of bias which affect the structure and variability of ENSO, including the SST cold tongue bias across the central equatorial Pacific, the zonal wind stresses of the atmosphere on the ocean, and model thermocline temperatures $[9,6]$ in the equatorial Pacific Ocean down to a depth of $300 \mathrm{~m}$.

The observed reanalysis SST forcings applied to PECOAM show annually 
averaged temperatures ranging from $20^{\circ} \mathrm{C}$ to $30^{\circ} \mathrm{C}$ with the maximum centred along the equator in the Pacific Ocean region. When we compare the SST forcings taken from both CGCMs against that of the Standard model, we observe anomalies ranging between $\pm 1^{\circ} \mathrm{C}$. Figure $4(\mathrm{~b})$ shows ACCESS 1-3 with the characteristic cold tongue bias along the equator, as described by Rashid et al. [9], while CCSM4 in Figure 4(c) has a reduced cold bias, but has wide spread ocean temperatures that are generally too warm. Increased ocean surface temperatures suggest more convective instability and a possible cause of model forecast error growth. A comprehensive description of the development of CCSM4 and its biases is given by Gent et al. [6], with a description of the annual cycle anomalies of SST along the equator in the Pacific Ocean shown in their Figure 7(c). They showed warmer than observed temperatures occuring through September, which may be a contributing cause to the secondary peak in Figure 1(b) of our CCSM4 forced PECOAM runs.

The annual cycle of the CGCM mean and shear atmospheric potential temperature climatology was also analysed (not shown) for comparison with NNR. Both models displayed shear temperatures approximately $1{ }^{\circ} \mathrm{C}$ above that of NNR in the equatorial region. Unlike ACCESS1-3, CCSM 4 annually averaged mean temperatures show large regions of warming between $30^{\circ} \mathrm{S}$ and $90^{\circ} \mathrm{S}$.

\section{Conclusion}

We have established a novel approach to examine the role of model bias in predicting ENSO using a primitive equation coupled ocean atmosphere model. The model reproduces the average annual cycle of climatology of the atmosphere and ocean based on forcing functions derived from the NNR data and ACCESS1-3 and CCSM 4 climate models during the twenty year period from 1980 to 1999. We employ an efficient cyclic mode method of breeding perturbations for our eight member ensemble forecasts and describe the seasonal variability of these fast growing coupled instabilities by calculating their average local total growth rates and relative amplification factors. 
Standard

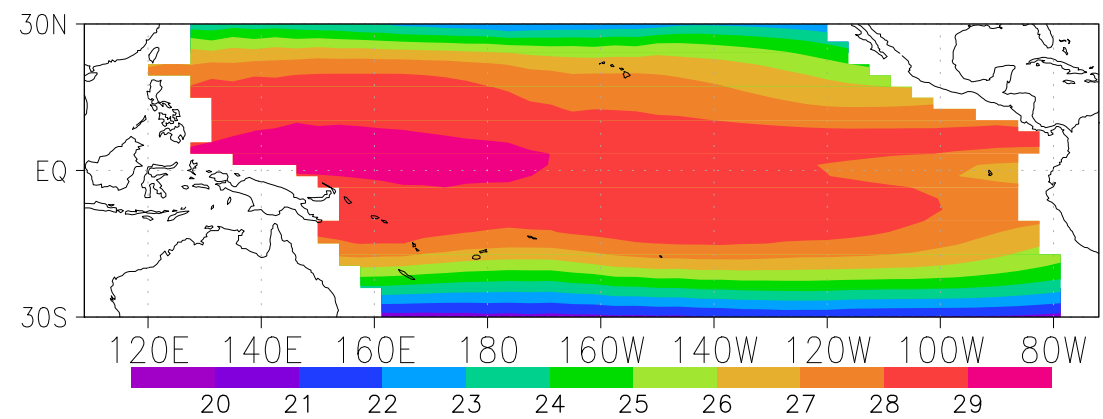

ACCESS 1.3

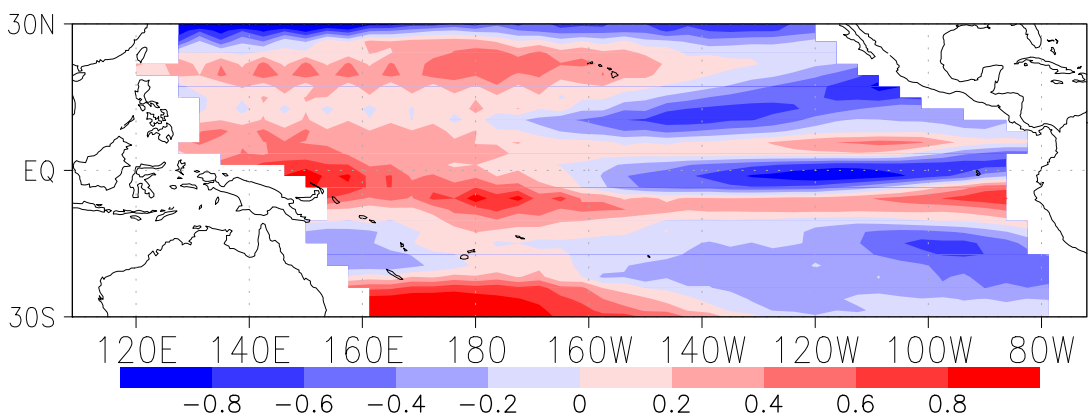

$\operatorname{ccsm} 4$

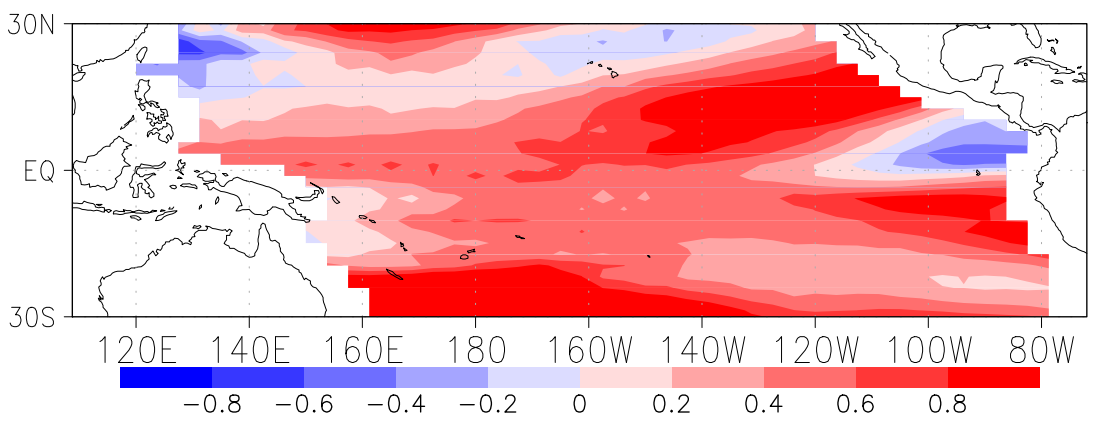

Figure 4: Annual averaged Sea Surface Temperatures $\left({ }^{\circ} \mathrm{C}\right)$ in the Pacific Ocean region between 1980 and 1999 for: (top) Standard; (middle) the departure of ACCESS1-3 values from Standard; and (bottom) the departure of CCSM4 values from Standard. 
This study has shown that seasonal ensemble prediction is sensitive to changes in the applied forcing functions and analyses. The PECOAM was unable to generate large ENSO events with the CGCM based forcings, as demonstrated by the underestimation of ocean temperature anomalies in the Pacific Ocean during the analysis runs. Other studies have shown that CGCM models are most skillful once large ENSO events are established, with minimal skill during neutral periods [1]. The ACCESS1-3 and, to a lesser extent, CCSM4 forcings applied to the PECOAM model are unable to generate large amplifying features of an extreme ENSO event thereby reducing the model's ability to produce skillful seasonal predictions. We also find that when the PECOAM is forced with either ACCESS1-3 or CCSM4 forcing functions that their average rate of error growth is larger than for the Standard configuration. A more unstable basic state and perturbed analyses suggest less reliable performance in seasonal forecasting of ENSO. The seasonal cycle variability of growth rates and relative amplification factors are both similar to the results produced by the Standard forcings, but with a secondary maxima occurring in September for CCSM4 forcings. The ensemble forecast shows improvement over the control forecast in almost all instances. The BSPB is evident in each of the three cases plus an increased error during forecasts initiated in the months of March-April-May, although less pronounced for PECOAM run with ACCESS1-3 forcings.

Gaining insight into how well these models capture realistic ENSO and being able to better understand its predictability may provide benefits for researchers looking to improve rainfall projections for Australian businesses and the community. We plan to extend our analysis to look for changes in ENSO under climate change in a high emissions scenario by using different climate forcing functions that will reproduce the future climate in model projections.

Acknowledgements This project is supported through funding from the Australian Government's National Environmental Science Programme. Support for this study was also provided by the Australian Research Council Centre of Excellence for Climate System Science. 


\section{References}

[1] C. S. Frederiksen, J. S. Frederiksen, and R. C. Balgovind. "Dynamic variability and seasonal predictability in an intermediate complexity coupled ocean-atmosphere model". In: Proceedings of the 16th Biennial Computational Techniques and Applications Conference, CTAC-2012. Vol. 54. ANZIAM J. 2013, pp. C34-C55. DOI: 10.21914/anziamj.v54i0.6296 (cit. on pp. C217, C218, C228).

[2] C. S. Frederiksen, J. S. Frederiksen, and R. C. Balgovind. "ENSO variability and prediction in a coupled ocean-atmosphere model". In: Aust. Met. Ocean. J. 59 (2010), pp. 35-52. URL: http://www . bom.gov .au/jshess/papers . php?year=2010 (cit. on pp. C217, C218).

[3] C. S. Frederiksen, J. S. Frederiksen, J. M. Sisson, and S. L. Osbrough. "Trends and projections of Southern Hemisphere baroclinicity: the role of external forcing and impact on Australian rainfall". In: Clim. Dyn. 48 (2017), pp. 3261-3282. DOI: 10.1007/s00382-016-3263-8 (cit. on p. C219).

[4] J. S. Frederiksen, C. S. Frederiksen, and S. L. Osbrough. "Methods of ensemble prediction for seasonal forecasts with a coupled ocean-atmosphere model". In: Proceedings of the 16th Biennial Computational Techniques and Applications Conference, CTAC-2012. Vol. 54. ANZIAM J. 2013, pp. C361-C376. DOI: 10.21914/anziamj.v54i0.6509 (cit. on pp. C217, C219, C224).

[5] J. S. Frederiksen, C. S. Frederiksen, and S. L. Osbrough. "Seasonal ensemble prediction with a coupled ocean-atmosphere model". In: Aust. Met. Ocean. J. 59 (2010), pp. 53-66. URL:

http://www . bom.gov . au/jshess/papers . php?year=2010 (cit. on pp. C217, C219, C220, C224). 
[6] P. R. Gent et al. "The community Climate System Model version 4". In:

J. Clim. 24 (2011), pp. 4973-4991. DOI: 10.1175/2011 JCLI4083.1 (cit. on pp. C218, C225, C226).

[7] S. Grainger, C. S. Frederiksen, and X. Zheng. "Assessment of modes of interannual variability of Southern Hemisphere atmospheric circulation in CMIP5 models". In: J. Clim. 27 (2014), pp. 8107-8125. DOI: 10.1175/JCLI-D-14-00251.1 (cit. on p. C219).

[8] E. Kalnay et al. "The NCEP/NCAR 40-year reanalysis project". In: $B$. Am. Meteorol. Soc. 77 (1996), pp. 437-472. DOI: 10.1175/1520-0477(1996)077<0437:TNYRP>2.0.C0;2 (cit. on p. C218).

[9] H. A. Rashid, A. Sullivan, A. C. Hirst, D. Bi, X. Zhou, and S. J. Marsland. "Evaluation of El Niño-Southern Oscillation in the ACCESS coupled model simulations for CMIP5". In: Aust. Met. Ocean. J. 63 (2013), pp. 161-180. DOI: 10.22499/2.6301.010 (cit. on pp. C218, C225, C226).

[10] K. E. Taylor, R. J. Stouffer, and G. A. Meehl. "An overview of CMIP5 and the experiment design". In: Bull. Am. Meteorol. Soc. 93 (2012), pp. 485-498. DOI: 10.1175/BAMS-D-11-00094.1 (cit. on p. C218).

\section{Author addresses}

1. S. L. Osbrough, CSIRO, Aspendale, Victoria 3195, Australia. Monash University, Clayton, Victoria 3800, Australia. mailto:stacey.osbrough@csiro.au orcid:0000-0001-6999-2405

2. J. S. Frederiksen, CSIRO, Aspendale, Victoria 3195, Australia. Monash University, Clayton, Victoria 3800, Australia.

3. C. S. Frederiksen, BOM, Docklands, Victoria 3000, Australia. CSIRO, Aspendale, Victoria 3195, Australia. 